\title{
Efficient Scheduling and Collision Reduction in Hybrid Cognitive Radio Network using SBP
}

\author{
Rathika.P.D ${ }^{a}$, Dr.S.Sophia ${ }^{b}$ \\ ${ }^{a}$ Assistant Professor, Department of ECE, Jay Shriram Group of Institutions, Tirupur 638660, India \\ ${ }^{b}$ Professor, Department of ECE, Sri Krishna College of Engineering and Technology, \\ Coimbatore 641008, India
}

\begin{abstract}
\section{Keywords: Cognitive Radio Network, Quality of Service, broadcasting, prioritizing INTRODUCTION}

Nowadays, Cognitive Radio Networks have been considered, investigated and planned in an energetic manner. But maximum works are focused on separate areas like Allocation, Sensing and Sharing of Spectrum, etc. In this paper, a HCRN with the subsequent characteristics of priority based scheduling based on centralized switching delay aware network and distributed broadcasting protocol to decrease collision and enhance scheduling is proposed. For developing Hybrid Cognitive Radio Networks (HCRN) lot of space is still available. The proposed HCRN is simulated and network quality of service is estimated in terms of packet delivery ratio, control overhead, throughput and broadcast delay.

Recent technology developments have led to extensive development in the technologies of wired and wireless networks. In Wireless technology case, the spectrum exploitation constantly remains a bottleneck. Previously, spectrum allocation was fixed and managed to ineffective usage of the same. Maximum spectrum in certain applications like TV distribution continued as spectrum holes or white spaces. It provided the solution of the dynamic spectrum allocation, which permits the certified users to share their spectrum to unlicensed users when they are not using it. Such capable technology with huge growth is the Cognitive Radio Network.

The important functions in a Cognitive Radio Network contain Spectrum Allocation, Sensing, Sharing and Scheduling. Various algorithms have been proposed for Spectrum allocations and scheduling. In all these algorithms, switching involved remains a major concern when the frequency range of the network is very high. The type of traffic handled by the network may also be delay sensitive. The hardware components may cause variable delay depending on the frequency of operation.

Overall, the efficiency of a scheduling algorithm designed for a CRN depends on the delay involved during a Cognitive User switches its frequency.

In modern structural design, optimum methods are emerged with a new design of paradigm which aims to increase the scarce radio spectrum utilization and play an important role in optimization process. For better optimization of structure genetic algorithms are used for efficient process. It is designed and developed for finding a solution among contradictory objectives. It became an important tool for strategic decision making and to review the interactions between cognitive radios for operating communication system. Cognitive radio is intended in enhance the radio frequency spectrum utilization.

In cooperative communication system, the bandwidth and same power of legacy wireless communication systems can increase the future wireless communication system data rate. The combination of cognitive radio and cooperative communication system will improve the performance of the wireless network. For efficient access of resource allocation in cooperative cognitive radio network $(C R N)$ is vital to meet the wireless networks challenges [Naeem, $\mathrm{M}$ et al, 2014].

In multiple carrier frequencies the digital data are encoding using the extensive method of OFDM and for application and wideband digital communication purposes the methods are developed. At low symbol rate the conventional modulation scheme is modulated for each sub-carrier. It maintains the total data rates in same bandwidth similar to schemes. In order to achieve signal-to-noise ratio improvement the inter symbol interference, time spreading and utilize echoes are eliminated by using the interval between symbols affordable.

In order to overcome the issues and limitation of the existing system the SBP approach is implemented in HCRN. The research paper is organized as follows: In Section 2 the survey related to the allocation of resources and scheduling algorithm are discussed. Section 3 is presented with the proposed algorithm and methods with its implementation. In Section 4, the evaluated results analysis of the performances and comparison of various algorithms are presented. Finally, conclusion of the research work with its performances is in Section 5.

\section{RELATED WORK}

In this section the related survey of scheduling and resource allocation decisions is discussed for the analysis of the work to propose an algorithm to improve the performances and to overcome the limits of existing work. Al Rawi, A.F et al, [2014] processed the capability to operate the adapted context through rate control, channel selection and possible power control. The best possible strategies are chosen for the techniques and the mathematical expressions are performed by using game theory. To achieve similar metrics or connection rate are estimated or evaluated by the utility functions. It provides a decision making process to guide the optimum process with multiple objectives and the results in optimal compliance [Khaled Ben Letaiefm and Wei Zhang, 2009]. 


$$
\begin{array}{r}
\text { ISSN 2321-807x } \\
\text { Jolume 13 Number4 } \\
\text { Journal of Advances in chemistry }
\end{array}
$$

The first study on switching delay was given by Didem Gozupek et al., [2013], in which they propose a $S^{2}$ DASA algorithm with a PU spectrum occupancy Model. Priority based scheduling is necessary to provide better QoS to real time data traffic. The BRACER protocol proposed by Yi Song et al., [2015] is a multipath broadcasting protocol which gives a collision avoidance scheme to improve the QoS parameters for Secondary User.

OFDM-Based Cognitive Radio (CR) Networks consider the methodology for a distributed resource allocation mechanism. This method allows management of resources between CR devices. It takes the best problem formulation from cooperative and competitive, and provides control balances between spectral efficiency (SE) of resource allocation. The results are confirming the efficient resource utilization and practically may be used in wireless cognitive networks [Tung Thanh Le; Dong-Seong Kim, 2014; Duy Trong Ngo et al, 2011].

For utilizing radio resources the cognitive radio and cooperative communication methods are capable. The system process with the same bandwidth [Li Yu et al, 2014] and power, in order to increases the performances of the wireless system cooperative communication system and cognitive radio are blending for performances improvement. It evolves the use of network parameters in cooperative cognitive radio network (CRN) [15].

Generally orthogonal frequency - division multiplexing (OFDM) is used for resources allocation to the sub channels and it also pre-allocate the resources based on the request [Rawat, D.B et al, 2014]. So it can't take decision based on situation. Due to this bit error rate will be increased and quality of the services will be reduced by television white noise in resource allocation.

The resource allocation scheme is based on the development of the stable matching to takes the preferences of users into account. To improve robustness $\epsilon$-stable resource allocation scheme is proposed. The results show the robust of the channel state information variation [Lu Lu et al, 2014].

The bandwidth-aware localized-routing algorithm is capable of choosing the routes from the available spectrum bands [Niyato, D.; Hossain, E, 2009]. A mixed-integer linear programming (MILP) is formulates the problem of the utilized process. It determines the possible pairs of the bandwidth to select the router and offers a better solution of closed optimal for performances of router. It achieves $50 \%$ utilized throughput of network.

The measurement spaces are obtained in a multidimensional model with sparse data in order to provide smooth transitions between observed values. The linearity is not defensible so this algorithm is used for the assumption issues of any regression. It generates prediction for accurate probability score of the target and required more space for model storage. In application inherent requirements are needed for considering all the conflicting design by the efficient methods of multi-objective optimization [S. Mangold et sl, 2005].

Most of the previous works [G. Cheng et al., 2007; I. Filippini et al, 2009] consider the channel switching delays only. They do not discuss the delays caused by frequency separation and concentrate only on routing and minimizing the distance.

In our Hybrid CRN, we have combined the PU Spectrum Occupancy Model Based on S²DASA Algorithm and incorporated the Priority based scheduling in the model. We have also used BRACER Protocol to avoid collision in a distributed environment and hence improved the throughput. The QoS Parameters of this proposed HCRN is studied by simulations and the improvements are shown.

\section{PROPOSED SBP IN HYBRID COGNITIVE RADIO NETWORK}

In this section, the proposed HCRN is a combination of three modules. They are $S^{2} D A S A$ algorithm, BRACER protocol and Priority based scheduling which can be defined as SBP. Each module has its own advantage which is discussed in the following sections and the combination leads to visible improvements in the QoS parameters.

\subsection{Network Architecture}

The network architecture considered here consists of number of secondary users (SUs) and two or more primary users (PUs). All these nodes are controlled by a centralized Cognitive Base Station (CBS). The primary work of the CBS is to sense if any of the PUs are not using their licensed band and allocate the same to SUs. In case of ad hoc networks, there may be more than one CBS involved and the SUs and PUs may get associated to different CBS at different times by means of frequency switching. Figure 1 shows the Centralized Cognitive Radio Network Architecture. 


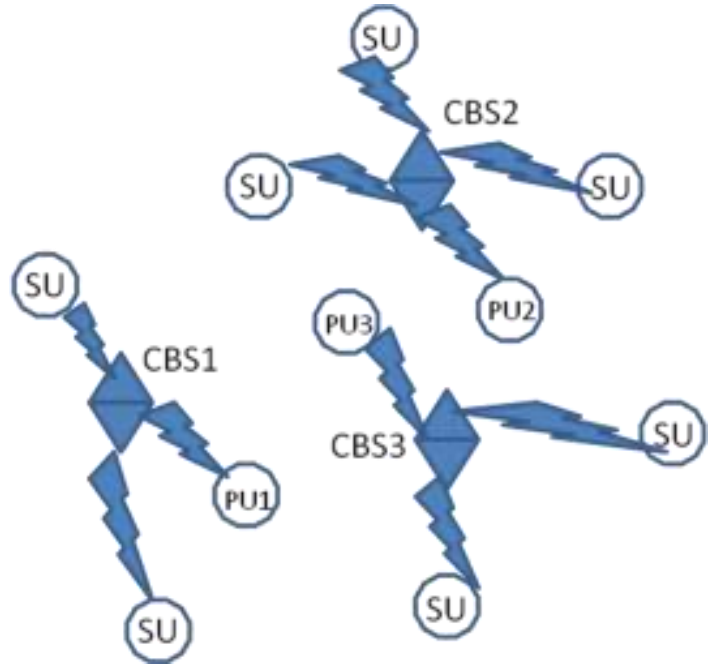

Fig 1: Centralized Cognitive Radio Network Architecture

\subsection{S'DASA Algorithm - $S$}

The finite state model for PU Spectrum occupancy is given in Figure 2. Each PU is either in ON or OFF state and can use any frequency out of $f$ available frequencies. Ps denotes the probability of PU staying in a particular state with frequency $f$ and (1-Ps) denotes the frequency of changing the frequency.

Here the nodes are considered to be moving and switching frequencies under following conditions.

- When the PU returns back to the licensed band

- When SU approaches another CBS

- If the SU is closer to another PU or SU physically causing interference

This switching should happen in a minimum guaranteed time so that the communication is not affected.

A Polynomial time heuristic algorithm can solve these switching delay problems in a CRN. The presence or absence of PUs is sensed by regular energy detection schemes. Whenever the CBS finds a free channel, it tries to allocate the same to one of the SUs. Now comes the frequency switching and delay involved with it.

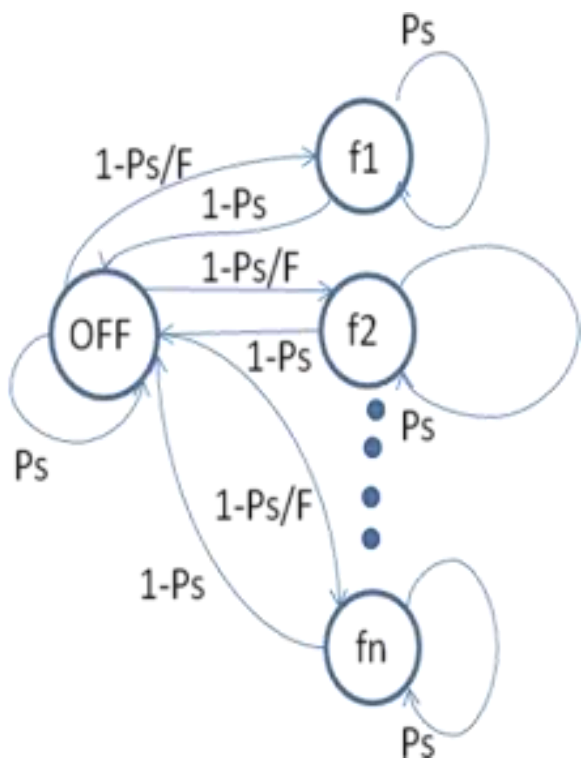

Fig 2: Finite State Spectrum Occupancy Model

By following the heuristic $S^{2} D A S A$ algorithm, CBS can allocate the free channel to an SU which has the least switching delay and can transmit maximum number of packets in the allocated time frame.

The $S^{2}$ DASA algorithm follows the following ILP: 
$\max \left(\sum_{i=1}^{N} \sum_{a=1}^{a i} \sum_{f=1}^{F} B_{i a f}^{\prime} X_{i a f}^{\prime}\right)$

Where, $\mathrm{B}^{\prime}{ }_{\text {iaf }}$ is the maximum number of packets sent through an SU interface; $X^{\prime}{ }_{i a f}=1$ if SU transmits using frequency $\mathrm{f} ; X^{\prime}$ iaf $=0$ otherwise; $a$ is the number of interfaces; $\mathrm{F}$ is the number of frequencies available and $\mathrm{f}$ is the frequency being used.

\subsection{BRACER Protocol - B}

The control messages in any adhoc network are normally sent as broadcast messages. In our HCRN, we make use of the BRACER protocol to improve the performance of the network by means of using a distributed broadcast scheduling scheme and collision avoidance scheme.

The SU nodes are assumed to know the location of all its two hop neighbors. Also, SUs are allowed to transmit within a scheduled time frame. Here broadcasting is done on selected channels of SUs by prior selection and hence saving bandwidth.

By using an intelligent distributed scheduling method of HCRN finds the shortest path for rebroadcasting and hence shortest broadcast delay. Collision during rebroadcasting is avoided by allotting different time slots for different intermediate nodes. Therefore, no two intermediate nodes will broadcast on the same channel simultaneously.

\subsection{Priority Based Scheduling - P}

The SUs are allowed to transmit in a particular time frame. Scheduling is done to tell which SU should transmit in which time frame. Here we consider a total of $\mathrm{N}$ sub channels with $\mathrm{f}$ frequencies. The number of sub channels available during one scheduling period is

$M=\frac{T_{S P}}{L}$

Where, $L$ is the length of the frame and $T_{S P}$ is the scheduling period. The data packets in the scheduler are segregated as real time and non real time and a priority value is set for them based on the equation as follows;

$P(\mathrm{i}, \mathrm{j})=\mathrm{c}_{j} \exp \left[\begin{array}{l}\alpha_{j}\left(\frac{W_{i j}(\mathrm{t})-T_{j}}{T_{j}}\right) \\ -\beta_{j}\left(\frac{b_{i j}}{R_{j} L}\right)\end{array}\right]$

Where, $c_{j}$ is the dynamic service coefficient, $R_{j}$ is the target bit rate, $T_{j}$ is the maximum packet delay bound, $\alpha_{j}$ and $\beta_{j}$ are the weights for balancing the impacts of the throughput and delay.

Also, $w_{i j}(t)$ is the waiting time for user I with traffic class $j . b_{i j}$ is the number of bits to be transmitted by user $i$ with traffic class j.

The Service coefficient can further be defined as

$c_{j}=1+e^{\frac{-N}{n}}$ If $\mathrm{j}$ is real time data traffic

$c_{j}=1 \quad$ If $\mathrm{j}$ is non-real time data traffic

\section{SIMULATION RESULTS}

In this section, the proposed system is simulated in network simulator assuming AWGN channels and random mobility. The PUs and SUs tend to switch cells and hence the frequency. The spectrum occupancy model of the $S^{2}$ DASA algorithm is followed. The communication between the CBS, PUs and SU are established using BRACER protocol. The data transmission is controlled by priority based scheduling. Some of the QoS parameters are studied and improvements are discussed below.

\subsection{Throughput}

Figure 3 shows the comparison of throughput between HCRN with other methods. Network throughput generally refers to the amount of bits successfully transmitted over a network. 


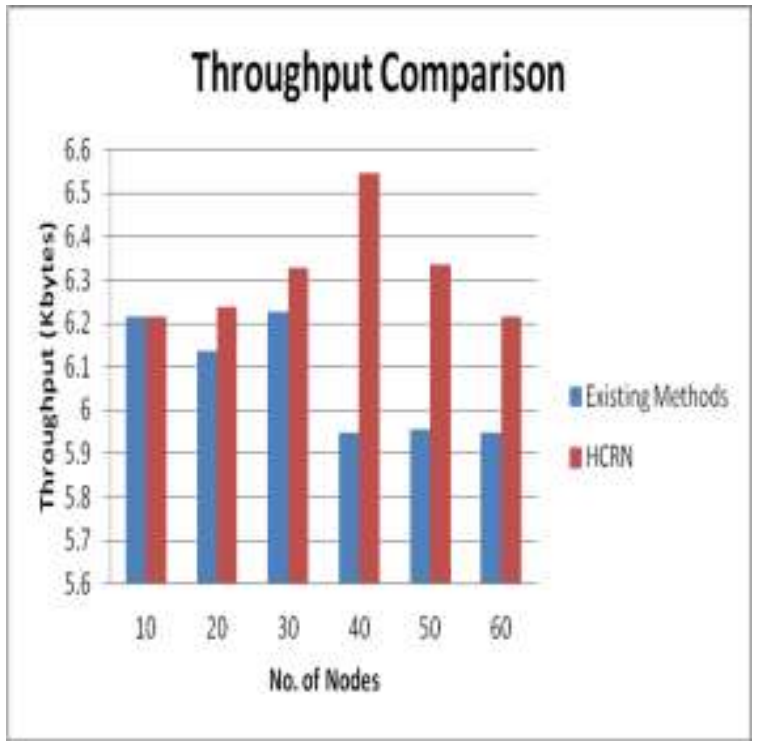

Fig 3: Comparison of Throughput

Here, the transmission includes communications between CBS to PU, PU to SU and CBS to SU. It is seen that the $S^{2}$ DASA algorithm makes sure that at least one time slot with a particular frequency $f$ is allocated to an SU if a PU is not using it.

Also, prioritizing the data into real time and non-real time reduces the queuing time. These factors lead to significant improvements in throughput of the proposed HCRN. The maximum throughput remains when the network has optimal number of nodes. The improvement here is about $10 \%$.

\subsection{Packet Delivery Ratio}

Packet Delivery Ratio is defined as the number of packets successfully delivered to a destination to the number of packets sent by the source.

Figure 4 shows the comparison of packet delivery ratio of HCRN with existing methods. It is clearly seen that HCRN outperforms other methods by about $5 \%$. The credit indeed goes to the perfect combination of $S^{2} D A S A$, BRACER and prioritizing.

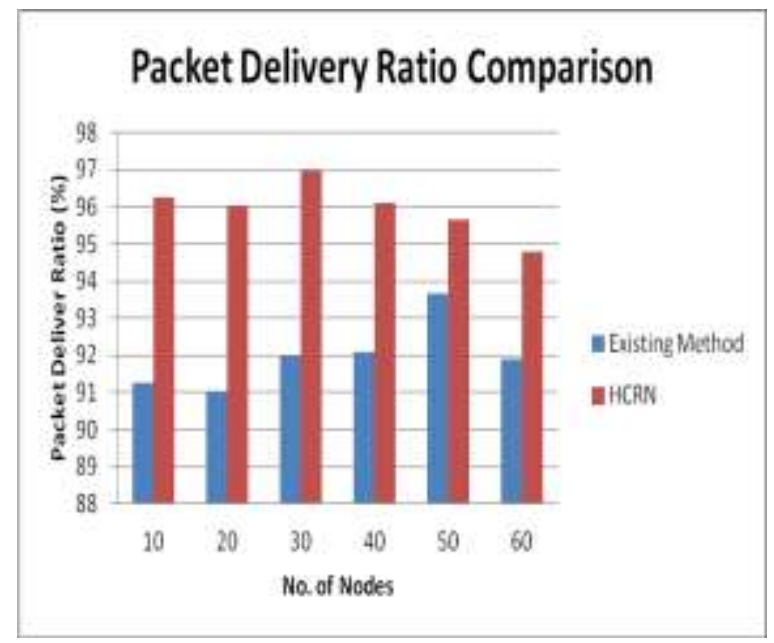

Fig 4: Comparison of Packet Delivery Ratio

\subsection{Broadcast Delay}

The delay increases as the number of nodes increase. But the proposed HCRN performs better than the other existing methods under any circumstance.

This is mainly due to the BRACER Protocol combined with prioritizing. The shortest paths assigned and the traffic handling proves to be effective in the proposed system.

Figure 5 shows the average broadcast delay for the proposed HCRN under various number of nodes. 


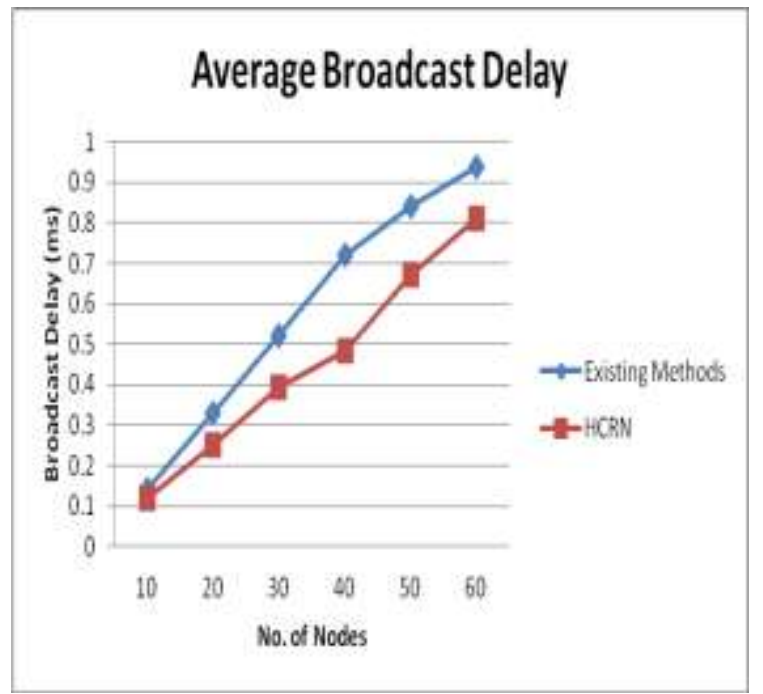

Fig 5: Comparison of Average Broadcast Delay

\subsection{Control Overhead}

Figure shows the comparison of normalized Control Overhead in the HCRN and the other existing methods. It is defined as the ratio of total number of packets broadcasted in the network to that received by the network.

The overhead here includes the information about neighbors, intermediate nodes required to rebroadcast and the original information. Also, it considered the variation of control overhead with respect to number of nodes in the network.

It is seen that the control overhead remains almost constant and lower for the proposed HCRN while it is slightly increasing in other existing methods. This is because of the smaller control information added to original packet length by means of BRACER protocol.

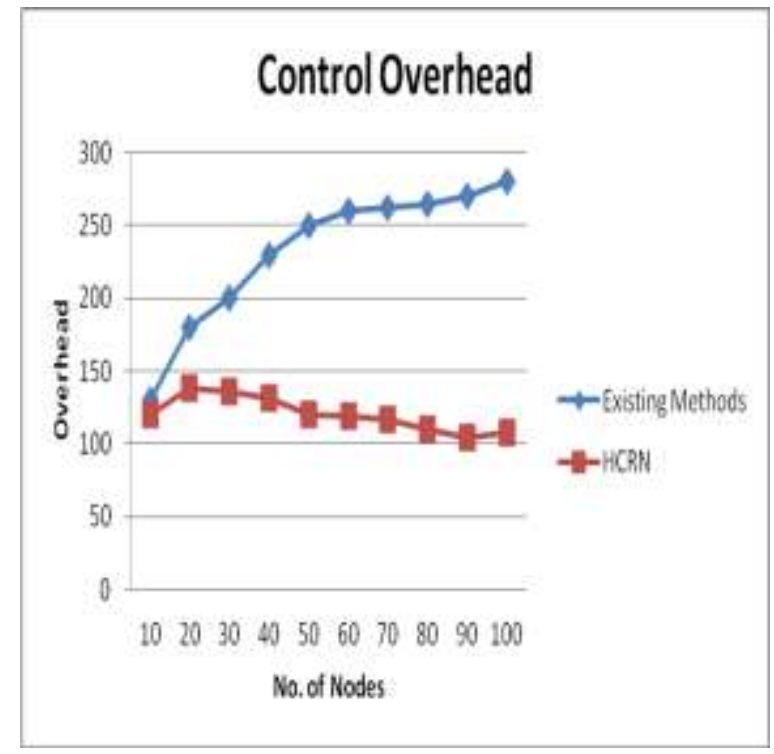

Fig 6: Comparison of Throughput

\section{CONCLUSION}

In this paper, hybrid cognitive radio network is proposed which incorporates the advantages of three various techniques and hence proved to be practically challenging and useful. The proposed system has been simulated as the hybrid network and studied the quality of service parameters. Also, it is able to achieve a better quality by intelligently combining various techniques. Hence, this network outperforms other existing methods. The evaluation of results demand schedule process provide with better performance than the existing scheme. The results can further be extended to the study of SNR, collision probability and efficiency of the network. 


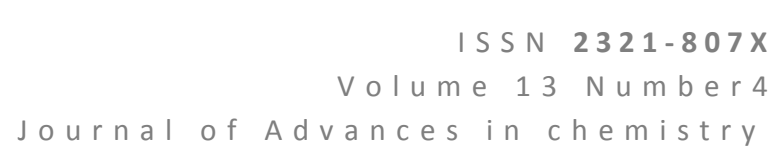

Furthermore, goal is to achieve an efficient resource allocation with less iteration time and better accuracy in access and allocation of bandwidth than the upcoming algorithm. Also plan to extend the work to improve the solutions for optimization issues in wireless network service.

\section{REFERENCES}

[1] Yi Song and Jiang XieBRACER, "A Distributed Broadcast Protocol in Multi-Hop Cognitive Radio Ad Hoc Networks with Collision Avoidance" IEEE Transactions on Mobile Computing, Vol 14, No. 3 , March 2015.

[2] Al Rawi, A.F.; Aissa, S.; Tsimenidis, C.C.; Sharif, B.S., "Game Theoretic Framework for Future Generation Networks Modelling and Optimization," Wireless Communications, IEEE Transactions on , vol.13, no.3, pp.1153,1163, March 2014 doi: 10.1109/TWC.2014.012314.120977

[3] Li Yu, Cong Liu, Wenwu Zhu, Sha Hua and Wei Wang, "Bandwidth Efficient and Rate-Adaptive Video Delivery in TV White Space", IEEE TRANSACTIONS ON CIRCUITS AND SYSTEMS FOR VIDEO TECHNOLOGY, VOL. 24, NO. 9, SEPTEMBER 2014, doi 10.1109/TCSVT.2014.2310135

[4] Lu Lu; Dawei He; Xingxing Yu; Li, G.Y., "Graph-based robust resource allocation for cognitive radio networks," Acoustics, Speech and Signal Processing (ICASSP), 2014 IEEE International Conference on , vol., no., pp.7298,7302, 4-9 May 2014 doi: 10.1109/ICASSP.2014.6855017

[5] Naeem, M.; Anpalagan, A.; Jaseemuddin, M.; Lee, D.C., "Resource Allocation Techniques in Cooperative Cognitive Radio Networks," Communications Surveys \& Tutorials, IEEE , vol.16, no.2, pp.729,744, Second Quarter 2014 doi: 10.1109/SURV.2013.102313.00272

[6] Rawat, D.B.; Shetty, S.; Raza, K., "Game Theoretic Dynamic Spectrum Access in Cloud-Based Cognitive Radio Networks," Cloud Engineering (IC2E), 2014 IEEE International Conference on , vol., no., pp.586,591, 11-14 March 2014 doi: 10.1109/IC2E.2014.16

[7] Tung Thanh Le; Dong-Seong Kim, "An efficient throughput improvement through bandwidth awareness in cognitive radio networks," Communications and Networks, Journal of, vol.16, no.2, pp.146,154, April 2014 doi: 10.1109/JCN.2014.000025

[8] Didem Go "zu "pek, Seyed Buhari, and Fatih Alagoz, "A Spectrum Switching Delay-Aware Scheduling Algorithm for Centralized Cognitive Radio Networks", IEEE Transactions on Mobile Computing, Vol. 12, No. 7, July 2013.

[9] Duy Trong Ngo; Le-Ngoc, Tho, "Distributed Resource Allocation for Cognitive Radio Networks With SpectrumSharing Constraints," Vehicular Technology, IEEE Transactions on , vol.60, no.7, pp.3436,3449, Sept. 2011 doi: 10.1109/TVT.2011.2157845

[10] Niyato, D.; Hossain, E., "Cognitive radio for next-generation wireless networks: an approach to opportunistic channel selection in ieee 802.11-based wireless mesh," Wireless Communications, IEEE , vol.16, no.1, pp.,, February 2009 doi: 10.1109 / MWC. 2009. 4804368.

[11] Filippini I, E. Ekici, and M. Cesana, "Minimum Maintenance Cost Routing in Cognitive Radio Networks," Proc. IEEE Sixth Int'I Conf. Mobile Adhoc and Sensor Systems (MASS), 2009.

[12] Khaled Ben Letaiefm and Wei Zhang, "Cooperative Communications for Cognitive Radio Networks" Proceedings of the IEEE | Vol. 97, No. 5, May 2009 doi: 10.1109/SURV.2014.021414.00066

[13] G. Cheng, W. Liu, Y. Li, and W. Cheng, "Spectrum Aware OnDemand Routing in Cognitive Radio Networks," Proc. IEEE Second Int'I Symp. New Frontiers in Dynamic Spectrum Access Networks (DySPAN), 2007.

[14] G. Cheng, W. Liu, Y. Li, and W. Cheng, "Joint On-Demand Routing and Spectrum Assignment in Cognitive Radio Networks," Proc. IEEE Comm. Int'l Conf. (ICC), 2007.

[15] S. Mangold, A. Jarosch, and C. Monney, "Cognitive Radio - Trends and Research Challenges," Swisscom Comtec Magazine 03/2005, pp. 6-9, July 2005.

\section{AUTHOR DETAILS}

Rathika.P.D is a Post Graduate in Communication Systems and is currently pursuing her PhD in Anna University,Chennai. She has nine years of experience in teaching and two years in Industry. She is currently working as an Assistant Professor at Jay Shriram Group of Institutions, Tirupur. Her research interests include wireless networks and signal processing.

S.Sophia is a Senior Professor in the Department of Electronics and Communications Engineering, Sri Krishna College of Engineering and Technology, Coimbatore. She has more than 15 years of teaching experience and has published many research papers, both in the national and international scenarios. Her research interests include wireless networks, wireless communication systems, signal processing and intelligent systems. 